\title{
Overweight dogs are more likely to display undesirable behaviours: results of a large online survey of dog owners in the UK
}

\author{
Alexander J. German ${ }^{1}$, Emily Blackwell ${ }^{2}$, Mark Evans ${ }^{3}$ and Carri Westgarth ${ }^{4}$ \\ ${ }^{1}$ Institute of Ageing and Chronic Disease, University of Liverpool, Neston, UK \\ ${ }^{2}$ School of Clinical Veterinary Science, University of Bristol, Langford, UK \\ ${ }^{3}$ Independent Veterinary Consultant, Guildford, UK \\ ${ }^{4}$ Institute of Infection and Global Health, University of Liverpool, Liverpool, UK
}

(Received 12 November 2016 - Final revision received 29 January 2017 - Accepted 8 February 2017)

Journal of Nutritional Science (2017), vol. 6, e14, page 1 of 6

doi:10.1017/jns.2017.5

Abstract

Much of the global canine population is now overweight, and this can adversely affect health, lifespan and quality of life. Undesirable behaviours are also common in pet dogs, and these can adversely affect welfare, as well as being stressful to owners. However, links between obesity and behavioural disorders have never previously been explored. An online survey was conducted between June and August in 2014, coinciding with the broadcast of a National UK television programme, exploring dog health, welfare and behaviour. Information gathered included signalment, overweight status and the prevalence of a range of undesirable behaviours. Fisher's exact test and OR were used to determine associations between overweight status and owner-reported behaviours. A total of 17028 responses were received. After data verification, the final dataset comprised 11154 dogs, $1801(16 \cdot 1 \%)$ of which were reported by owners to be overweight. Owners of overweight dogs were more likely to see them as 'a baby' $(P<0.0001)$ and allow them to sleep on their bed $(P<0.0001)$. Overweight dogs were also more likely to guard food $(P<0.0001)$ and steal food $(P<0.0001)$. Other undesirable behaviours more commonly reported in overweight dogs included barking, growling or snapping at strangers $(P=0 \cdot 0011)$ and other $\operatorname{dogs}(P=0 \cdot 0015)$, being fearful of outdoors $(P<0 \cdot 0001)$, and not always coming back when called $(P=0 \cdot 0011)$. Finally, owners were more likely to report that unsociable behaviours adversely affected their dog's health $(P<0 \cdot 0001)$. Overweight status is associated with a number of undesirable behaviours in dogs. Further studies are now required to explore the reasons for these associations.

Key words: Welfare: Obesity: Canine nutrition: Anxiety: Aggression: Fearfulness

A multitude of problems can affect the welfare of dogs, ranging from inappropriate husbandry to euthanasia of healthy dogs. In a recent study, seven experts were asked to rank welfare priorities in dogs, and two of the top-rank priorities were undesirable behaviours and obesity ${ }^{(1)}$. Undesirable behaviours are known to be common in pet dogs, with one study estimating $90 \%$ of the population to be affected ${ }^{(2)}$. Problems can include aggression to familiar people, strangers or other dogs; fearfulness; separation anxiety; and food-related behaviours such as food guarding, food stealing and coprophagia ${ }^{(3)}$.
Obesity is also a considerable welfare concern in dogs; recent studies suggest that approximately half of all pet dogs are overweight $^{(4,5)}$, and this can predispose dogs to many diseases ${ }^{(4,6,7)}$, poorer quality of life ${ }^{(8)}$ and a shorter lifespan ${ }^{(9)}$.

Whilst obesity and undesirable behaviours both have an impact on canine welfare, the degree to which they may be related is not known. Therefore, the aim of the present study was to examine the associations between overweight status and undesirable behaviours, based upon attitudes of owners in a large, anonymous, online survey.

* Corresponding author: A. J. German, fax +44 151795 6101, email ajgerman@liverpool.ac.uk 


\section{Methods and materials}

\section{Study design and methodology}

Over the summer of 2014, a television documentary series entitled 'Dogs - Their Secret Lives' aired on Channel 4 television. The four-part series dealt with the health and wellbeing of dogs in the UK, and aimed to inform the general public of issues of critical current importance. The series featured three of the study authors (A. J. G., E. B., M. E.). As part of the overall project, an online survey into the health and welfare of dogs was conducted between June and August in 2014, coinciding with the broadcast of a national UK television programme, exploring dog health, welfare and behaviour. The study was approved by the University of Liverpool Ethics Committee. Participation was voluntary, with owners wishing to participate logging onto the Channel 4 website in order to complete the survey. Owners gave their permission for their data to be used, in a fully anonymised form (i.e. no client-identifying data), and for the results to be publicised both on the television shows and online. Further, they were not required to answer questions that they were unclear about, or did not wish to answer. To be eligible for inclusion in the data analysis part of the study, dogs had to be adult $(\geq 2$ years of age) and questionnaire information needed to be complete, i.e. all questions used in the present study needed to be answered.

\section{Survey design}

The online survey comprised forty-three questions, four of which covered personal data and these were not shared with the study investigators. Other questions covered details of signalment (age, sex, neuter status, breed), body weight and overweight status (based upon the response to the survey question: 'Is your dog overweight?'), current body weight, lifestyle, questions on a range of undesirable behaviours, and activity. Free text boxes were available for the questions on age and body weight; the remaining questions were either binary in nature (yes/no) or categorical, and selections were made either by checking a box or selecting responses from a drop-down menu. Owners could only select one category. For overweight status, owners responded to the question, 'Is your dog overweight?', with their answer being based on their own subjective impression (i.e. no reference to a formal body condition scoring system). The main questions considered in the present study were those relating to undesirable behaviours, whereas the questions involving activity are reported in two associated studies ${ }^{(10,11)}$

\section{Data handling and statistical analysis}

All data were collated into a computer spreadsheet (Excel version 14; Microsoft) for analysis. Initially, data cleaning was conducted to ensure reliability of the data analysed. First, given that body weight was used to confirm overweight status (see below), it was necessary to ensure that growing dogs were not included in the dataset. This was done by removing data from all dogs under 2 years of age. The dataset was further cleaned by removing dogs with any missing data, and dogs with obvious errors in the dataset for age and body weight. The latter involved manually checking the spreadsheet for obvious data errors. First, the entire spreadsheet was inspected for obvious erroneous values (e.g. age or body weight entered as 0 or an improbable value, i.e. age $>30$ years, body weight $>150 \mathrm{~kg}$ ). Next, age data were sorted first by breed and then by age using the 'sort' function. For each breed, individual dog ages were checked and compared with expected age range of the age of the breed based upon that reported within an online encyclopaedia (https://www.wikipedia.org). Dogs with ages more than $20 \%$ outside the range reported for each breed (given uncertainties of the reported age ranges) were removed. Finally, body weight data were sorted first by breed, then by sex, and then overweight status. Dogs that were reportedly not overweight but had a body weight more than $20 \%$ above the range reported for each breed were removed.

Computer software (Stats Direct version 3.0.171; Stats Direct Ltd) was used for all tests. Data are expressed as median (range) unless otherwise stated. OR and $95 \%$ CI were calculated in order to determine the association between owner-reported overweight status and various parameters, including sex, neuter status, and the responses to unsociable behaviours. Exceptions were for comparisons of age and body weight, where the MannWhitney test was used, and for the question how do you see your dog?', which had multiple categories and was assessed with Fisher's exact test. First, the test was performed across all categories simultaneously using the ' $\mathrm{r}$ by c Fisher' function in Stats Direct. Subsequently, the proportion of owners that selected the category 'baby' was compared with the proportion of owners selecting another answer using a $2 \times 2$ Fisher's exact test. Given that multiple comparisons were performed, a modified Bonferroni correction was applied ${ }^{(12)}$. This correction effectively meant that statistical significance was only considered when $P<0 \cdot 0017$.

\section{Results}

\section{Final dataset}

A total of 17028 responses were received. After cleaning, the final dataset comprised 11154 dogs, 6220 of which were male (4932 neutered; $79.3 \%$ ) and the remaining 4934 were female (4280 neutered; $86.8 \%$ ). Over eighty breeds were represented, with the most common being Border collie (583), Cavalier King Charles spaniel (224), cocker spaniel (512), mixed breed (2794), English springer spaniel (473), German shepherd dog (336), golden retriever (276), Jack Russell terrier (606), Labrador retriever (1344), Staffordshire bull terrier (451) and West Highland white terrier (217). Median age was 5 years (range 2-19 years) and median body weight was $20 \mathrm{~kg}$ (range 1-107 kg).

\section{Owner-reported overweight status}

Of the 11154 dogs, 1801 dogs were reported to be overweight $(16 \cdot 1 \%)$. To confirm that the overweight population was representative, associations with various signalment variables were 
assessed. Overweight dogs were significantly older (6 (range 216) years $v .5$ (range 2-19) years; $P<0.001$ ) and heavier (22 (range 1-107) kg v. 20 (range 1-107) kg; $P<0 \cdot 0001$ ) than dogs that were reported to be of ideal weight. Overweight status was also positively associated with being neutered, but not with sex, whilst a range of breeds was either positively (beagle, Cavalier King Charles spaniel, Chihuahua, Labrador retriever and pug) or negatively (German shepherd dog and greyhound breeds) associated (Table 1).

\section{Associations between owner attitudes and overweight status}

When owners were asked how they viewed their dog, those that owned overweight dogs were more likely to select 'your baby' (507/2548 (20\%) overweight; OR 1.538; $P<0.0001)$ than to use one of the other descriptions (total 1294/8606 (15\%) overweight: 'your protection', 1/35 (3\%); 'your assistant', 3/36 (8 $\%)$; 'your companion', 570/3812 (15\%); 'your best friend', $339 / 2089(16 \%)$ or 'your pet', $381 / 2634(14 \%))$. Owners of overweight dogs were also more likely to allow them to sleep in or on their bed (OR 1.432; $95 \%$ CI 1.293, 1.539; $P<$ $0 \cdot 0001)$, but not more likely to keep a photograph of their pet with them (OR 1.034; $95 \%$ CI 0.927, 1.153; $P=0.5522$ ).

\section{Associations between overweight status and undesirable behaviours}

Associations between overweight status and a range of undesirable behaviours were tested (Table 2). Dogs that were reported to be overweight were more likely to guard food $(P<0 \cdot 0001)$, steal food $(P<0 \cdot 0001)$, not always come back when called $(P=0.0002)$, be fearful or reluctant to walk outside $(P<0.0001)$, and be more likely to bark/ growl/snap at other dogs $(P=0 \cdot 0015)$ and strangers $(P=$ 0.0011). Dogs that did not always come back when called were less likely to be let off the lead than those that always returned (OR 0.425; $95 \%$ CI 0.388, 0.465; $P<0.0001$ ). Similar associations were also seen between dogs barking, growling at both other dogs (OR 0.410; $95 \%$ CI 0.374, $0.449 ; P<0.0001$ ) and strangers (OR 0.650; $95 \%$ CI 0.581, $0.727 ; P<0.0001)$ and the likelihood of being let off the lead. However, a direct association between overweight status and being let off the lead was not identified (OR 0.908; $95 \%$ CI $0.807,1.023 ; P=0.1060)$. Further, there was no association with a range of other behaviours (Table 2).

\section{Influence of undesirable behaviour and health}

More owners of overweight dogs reported that behavioural issues adversely affected health (752/1801, $42 \%$ ) than for owners who did not believe their dog to be overweight (3347/9353, $36 \%$; OR 1.286; $95 \%$ CI 1.159, 1.427; P<0.0001).

\section{Discussion}

This study describes the largest survey of owner attitudes to canine behaviour and welfare ever conducted, and some intriguing associations were identified between obesity and undesirable behaviour, including for the food-related behaviours (guarding and stealing food), displays of aggression to strangers or other dogs, being fearful of walking, and not returning when called. Furthermore, owners of overweight dogs were more likely to report that such undesirable behaviours were more likely to affect health than did owners of dogs not reported to be overweight. Given the fact that the study was cross-sectional in nature, the reasons for these associations cannot be determined, including whether or not they are causal. Prospective studies should now be considered to explore the nature of these associations more completely.

Assuming that the associations discovered are genuine, the link between overweight status and both food-seeking and food-guarding behaviour is perhaps most logical. For example, there might be common risk factors for both, for example that cause a stronger drive for food, making affected dogs more likely both to display food-related behaviours and to overeat causing weight gain. Such factors could be genetic and, in this regard, a deletion in the canine pro-opiomelanocortin gene was discovered in some dogs of the Labrador retriever breed, and this was found to be associated with both appetite and weight status in affected $\operatorname{dog} \mathrm{s}^{(13)}$. Alternatively, the behaviour of the owner might be important, whereby different styles of ownership might mean different feeding regimens, food types and rewards, predisposing some dogs to weight gain and undesirable food-related behaviour. Here, a parallel can be drawn with parenting and childhood obesity. In this respect, different parenting styles have been identified which reflect differences in the degree of control the parent places over their child's behaviour and the responsiveness of the parent to their child's wishes ${ }^{(14)}$. The children of parents who display either an indulgent style (i.e. displaying warmth and respect for their child's needs but only limited monitoring of their behaviour) or an authoritarian style (i.e. making high demands on their children whilst showing little responsiveness to their opinions or wishes) are more likely to be overweight that those who show other styles ${ }^{(14,15)}$. There are many parallels between how parents care for children and how owners care for their dogs, and a recent review considered how pet ownership styles could be mapped to parenting styles ${ }^{(16)}$. If dog-ownership styles exist that are similar to the indulgent and authoritarian parent styles, then similar predispositions might exist for canine obesity. Whilst the present work did not examine this directly, it was notable that owners who reported their dog to be overweight appeared to have a different relationship with them, in that they were more likely to see them as a baby and more likely to let them sleep in or on the bed. Further, other studies have demonstrated that owners of overweight dogs feed them more snacks and table scraps, observe them more closely during feeding and also more often allow the $\operatorname{dog}$ to be present when preparing a meal $^{(17,18)}$. Additional studies could examine attitudes and opinions of owners towards feeding their dogs, and explore the similarity to parenting behaviour more fully.

Possible reasons for associations between being overweight and other undesirable behaviours are less clear. It is possible that owners whose dogs demonstrate aggressive behaviours to strangers and other dogs are less likely to exercise them 
Table 1. Association between signalment factors and overweight (OW) status (Number of dogs; odds ratios and $95 \%$ confidence intervals)

\begin{tabular}{|c|c|c|c|c|c|}
\hline Variable & OW & Not OW & OR & $95 \% \mathrm{Cl}$ & $P+$ \\
\hline Age (per year) & - & - & 1.076 & $1.061,1.092$ & $<0.0001^{*}$ \\
\hline \multicolumn{6}{|l|}{ Sex } \\
\hline Female (reference) & 1583 & 8165 & 1.000 & - & - \\
\hline Male & 218 & 1188 & 0.946 & $0.807,1.06$ & 0.509 \\
\hline \multicolumn{6}{|l|}{ Neuter status } \\
\hline Entire (reference) & 177 & 1765 & 1.000 & - & - \\
\hline Neutered & 1624 & 7588 & $2 \cdot 134$ & $1 \cdot 810,2 \cdot 19$ & $<0.0001^{*}$ \\
\hline Body weight (per kg) & - & - & 1.018 & $1.014,1.022$ & $<0.0001^{*}$ \\
\hline \multicolumn{6}{|l|}{ Breed } \\
\hline Bassett & 12 & 36 & 1.734 & $0.901,3.343$ & 0.099 \\
\hline Beagle & 57 & 52 & 5.846 & $4.000,8.543$ & $<0.0001^{*}$ \\
\hline Bichon frise & 14 & 60 & 1.213 & $0.677,2.176$ & 0.516 \\
\hline Border collie & 74 & 509 & 0.744 & $0.580,0.955$ & 0.020 \\
\hline Border terrier & 37 & 162 & $1 \cdot 190$ & $0.830,1.707$ & 0.345 \\
\hline Boxer & 22 & 141 & 0.808 & $0.514,1.270$ & 0.355 \\
\hline Bull terrier & 12 & 36 & 1.734 & $0.901,3.343$ & 0.099 \\
\hline Bulldog & 14 & 30 & 2.435 & $1.288,4.600$ & 0.0061 \\
\hline Cairn terrier & 14 & 41 & 1.779 & $0.968,3.271$ & 0.064 \\
\hline Cavalier King Charles spaniel & 67 & 157 & $2 \cdot 263$ & $1.692,3.026$ & $<0.0001^{*}$ \\
\hline Chihuahua & 29 & 63 & 2.413 & $1.550,3.757$ & $<0.0001^{*}$ \\
\hline Cocker spaniel & 102 & 410 & 1.310 & $1.048,1.637$ & 0.018 \\
\hline Dachshund & 20 & 75 & 1.389 & $0.846,2.281$ & 0.194 \\
\hline Dalmatian & 5 & 66 & 0.392 & $0.158,0.974$ & 0.044 \\
\hline Doberman & 9 & 51 & 0.916 & $0.450,1.884$ & 0.809 \\
\hline English springer spaniel & 63 & 410 & 0.791 & $0.603,1.036$ & 0.088 \\
\hline Flat-coated retriever & 3 & 46 & 0.338 & $0.105,1.087$ & 0.069 \\
\hline German shepherd dog & 31 & 305 & 0.520 & $0.358,0.754$ & $0.0006^{*}$ \\
\hline Golden retriever & 62 & 214 & 1.522 & $1.142,2.029$ & 0.0041 \\
\hline Greyhound & 9 & 182 & 0.253 & $0.129,0.495$ & $<0.0001^{*}$ \\
\hline Hungarian Vizla & 4 & 51 & 0.406 & $0.146,1.125$ & 0.083 \\
\hline Irish setter & 2 & 38 & 0.272 & $0.066,1.131$ & 0.073 \\
\hline Jack Russell terrier & 99 & 507 & 1.015 & $0.813,1.266$ & 0.896 \\
\hline Labrador retriever & 285 & 1059 & 1.472 & $1.278,1.697$ & $<0.0001^{*}$ \\
\hline Lhasa apso & 17 & 53 & 1.672 & $0.966,2.894$ & 0.066 \\
\hline Miniature Schnauzer & 25 & 95 & 1.372 & $0.880,2.137$ & 0.162 \\
\hline Poodle & 3 & 76 & 0.204 & $0.064,0.646$ & 0.0069 \\
\hline Pug & 17 & 22 & 4.042 & $2 \cdot 142,7.626$ & $<0.0001^{*}$ \\
\hline Rhodesian ridgeback & 7 & 37 & 0.982 & $0.437,2.207$ & 0.966 \\
\hline Rottweiler & 18 & 86 & 1.088 & $0.653,1.812$ & 0.746 \\
\hline Rough-coated collie & 11 & 53 & 1.078 & $0.562,2.068$ & 0.820 \\
\hline Shih Tzu & 19 & 83 & $1 \cdot 191$ & $0.721,1.965$ & 0.494 \\
\hline Siberian husky & 7 & 61 & 0.594 & $0.271,1.301$ & 0.193 \\
\hline Staffordshire bull terrier & 85 & 366 & 1.212 & $0.955,1.549$ & 0.112 \\
\hline Weimaraner & 5 & 47 & 0.551 & $0.219,1.388$ & 0.206 \\
\hline West Highland white terrier & 41 & 176 & 1.215 & $0.861,1.713$ & 0.267 \\
\hline Whippet & 5 & 68 & 0.380 & $0.153,0.944$ & 0.037 \\
\hline Yorkshire terrier & 19 & 96 & 1.028 & $0.627,1.687$ & 0.912 \\
\hline
\end{tabular}

* Results reaching statistical significance.

† Modified Bonferroni correction applied, so statistical significance considered when $P<0.0017$.

outside or restrict their freedom if they $\mathrm{do}^{(19)}$. Indeed, we found that such dogs were less likely to be let off the lead than those not displaying such behaviours. However, given that there was no direct association between dogs being let off the lead and overweight status, other factors might also contribute. One alternative reason for the link between undesirable behaviours and overweight status may be the incorrect practice of reward-based behaviour therapy/dog training, in terms of excessive food rewards to reinforce positive behaviour, thereby encouraging overeating. For this reason, owners should always be recommended to adjust the dog's food intake appropriately and ideally use the premeasured daily food ration for training.
One final observation was the fact that owners of overweight dogs were more likely to state that undesirable behaviours adversely affected their dog's health. The reason for this association is not clear. It might be a direct association, for example the overweight status making the behaviour more severe. Alternatively, the association might represent a cumulative effect on health. In this respect, obesity is known to adversely affect quality of life in $\operatorname{dogs}{ }^{(8)}$, so it might appear that dogs also displaying an undesirable behaviour are perceived to have a greater adverse effect on health simply because the dog is starting from a lower baseline quality of life. Once again, further studies are required both to confirm this association and to explore the reasons for it. 
Table 2. Association between behaviours and overweight status (Proportion and percentage of overweight dogs in each response category; odds ratios and $95 \%$ confidence intervals)

\begin{tabular}{|c|c|c|c|c|c|c|c|}
\hline \multirow{2}{*}{$\begin{array}{l}\text { Which of the following behaviours does your dog } \\
\text { display on a regular basis? }\end{array}$} & \multicolumn{2}{|l|}{ Yes } & \multicolumn{2}{|l|}{ No } & \multirow[b]{2}{*}{ OR } & \multirow[b]{2}{*}{$95 \% \mathrm{Cl}$} & \multirow[b]{2}{*}{$P+$} \\
\hline & $n$ & $\%$ & $n$ & $\%$ & & & \\
\hline Eat faeces & $364 / 1993$ & $18 \cdot 3$ & $1437 / 9161$ & $15 \cdot 7$ & $1 \cdot 201$ & $1.055,1.365$ & 0.0053 \\
\hline Guard food items/possessions & $261 / 1151$ & $22 \cdot 7$ & $1540 / 10003$ & $15 \cdot 4$ & 1.612 & $1.384,1.872$ & $<0.0001^{*}$ \\
\hline Steal food or scavenge items & $617 / 2765$ & $22 \cdot 3$ & $1184 / 8389$ & $14 \cdot 1$ & 1.748 & $1.565,1.951$ & $<0.0001^{*}$ \\
\hline Bark, howl, destroy, toilet or chew things at home & $174 / 1148$ & $15 \cdot 2$ & $1627 / 10006$ & $16 \cdot 3$ & 0.920 & $0.772,1.092$ & 0.3517 \\
\hline Toilet indoors when home alone & $62 / 334$ & $17 \cdot 6$ & $1739 / 10820$ & $16 \cdot 1$ & $1 \cdot 190$ & $0.884,1.582$ & $0 \cdot 2270$ \\
\hline Bark/whine when not home alone & $305 / 1733$ & $17 \cdot 6$ & $1496 / 9421$ & $15 \cdot 9$ & $1 \cdot 131$ & $0.985,1.297$ & 0.0758 \\
\hline \multicolumn{8}{|l|}{ Avoid/run away from } \\
\hline Other people & $148 / 874$ & $16 \cdot 9$ & $1653 / 10280$ & $16 \cdot 1$ & 1.064 & $0.879,1.282$ & 0.5029 \\
\hline Other dogs & 233/1378 & $16 \cdot 9$ & $1568 / 9776$ & $16 \cdot 0$ & 1.065 & $0.912,1.240$ & 0.4117 \\
\hline Not always come back when called & $842 / 4771$ & $17 \cdot 6$ & $959 / 6383$ & $15 \cdot 0$ & $1 \cdot 212$ & $1.093,1.343$ & $0.0002^{*}$ \\
\hline Pull on lead & $869 / 5534$ & $15 \cdot 4$ & $932 / 5520$ & $16 \cdot 9$ & 0.898 & $0.810,0.994$ & 0.0371 \\
\hline Jump up or try to get attention from people & $798 / 5086$ & $15 \cdot 7$ & $1003 / 6068$ & $16 \cdot 5$ & 0.940 & $0.848,1.041$ & 0.2348 \\
\hline \multicolumn{8}{|l|}{ Bark growl snap at } \\
\hline Other dogs & $629 / 3535$ & $17 \cdot 8$ & $1172 / 7619$ & $15 \cdot 4$ & 1.191 & $1.069,1.326$ & $0.0015^{\star}$ \\
\hline Strangers & $346 / 1847$ & $18 \cdot 7$ & $1455 / 9307$ & $15 \cdot 6$ & 1.244 & $1.089,1.418$ & $0.0011^{*}$ \\
\hline Familiar people & $114 / 536$ & $21 \cdot 3$ & $1687 / 10618$ & $15 \cdot 9$ & 1.344 & $1.076,1.668$ & 0.0078 \\
\hline \multicolumn{8}{|l|}{ Chase } \\
\hline Traffic & $91 / 523$ & $17 \cdot 4$ & $1710 / 10631$ & $16 \cdot 1$ & 1.099 & $0.862,1.389$ & 0.4288 \\
\hline Other things like cyclists, joggers & $188 / 1057$ & $17 \cdot 8$ & $1613 / 10097$ & $16 \cdot 0$ & $1 \cdot 138$ & $0.958,1.346$ & 0.1352 \\
\hline Fearful of noises - run away, hide, whimper & $188 / 1057$ & $17 \cdot 8$ & $1613 / 10097$ & $16 \cdot 0$ & 1.018 & $0.896,1.156$ & 0.7726 \\
\hline Fearful of outside - reluctant to walk & $65 / 254$ & $25 \cdot 6$ & $1736 / 9484$ & $15 \cdot 9$ & 1.815 & $1 \cdot 341,2.431$ & $<0.0001^{\star}$ \\
\hline Obsessively lick himself/herself or people & $304 / 1670$ & $18 \cdot 2$ & $1497 / 9484$ & $15 \cdot 8$ & 1.187 & $1.0328,1.362$ & 0.0142 \\
\hline Spin or chase his/her tail & $109 / 857$ & $12 \cdot 7$ & $1692 / 10297$ & $16 \cdot 4$ & 0.741 & $0.596,0.914$ & 0.0043 \\
\hline $\begin{array}{l}\text { Have the selected behaviours affected your } \\
\text { dog's health or happiness? }\end{array}$ & $752 / 4099$ & $18 \cdot 3$ & $1049 / 7055$ & 14.9 & $1 \cdot 286$ & $1.159,1.427$ & $<0.0001^{*}$ \\
\hline
\end{tabular}

* Results reaching statistical significance.

† Modified Bonferroni correction applied, so statistical significance considered when $P<0.0017$.

The study has a number of limitations that should be considered. Most notable was the fact that only $16 \cdot 1 \%$ of dogs were reported to be overweight by their owner, which is considerably less than current estimates of overweight and obesity in UK $\operatorname{dogs}{ }^{(5)}$. The reason for this is not clear. One explanation might be participation bias, whereby the owners who were willing to participate were not representative of the UK dog-owning population. For example, dog owners could have been reluctant to participate if they knew their dog was overweight or were concerned. A second possible explanation for the low prevalence of overweight dogs might be misinterpretation of body shape by owners, since recent studies have demonstrated that owners underestimate the true body condition of their dog, often not realising they are overweight ${ }^{(20)}$. This is a concern for the present study because owners were not given any criteria to use for deciding whether their dog was overweight. The upshot of this would be many overweight dogs being inappropriately classified as not overweight, and this would tend to obscure differences between groups. Therefore, the true effect size of the associations between overweight status and undesirable behaviours might actually be stronger than was seen in the current study. Ideally, therefore, the current findings should now be confirmed with studies using different methods of assessing overweight status, for example the use of body condition scoring conducted by a trained veterinary professional.

A second limitation was the fact that the use of an online format meant that response options were limited, with owners having to choose from categories or binary options (i.e. yes or no). Therefore, there was no opportunity to explore the observations in any more detail, and the fact that the survey was fully anonymised means that further information cannot be obtained from the owners who participated. Third, given that the survey was conducted in association with a television documentary series examining behaviour and obesity in dogs, results might have been influenced by response bias, with the effect that owners consciously or subconsciously adapted their responses having watched the programmes.

Finally, this study is limited by the fact that it is exploratory in nature, and only examined simple associations without adjustment for confounding. This was not undertaken because of the multiple comparisons that had already been performed. It is likely that behavioural variables may correlate with confounders such as reduced exercise, or breed, which also lead to overweight. Future studies purposefully designed to examine independent associations should use multivariable analyses to adjust for confounding variables.

\section{Conclusions}

In the present study, overweight status in dogs (as reported by their owners) was associated with a number of undesirable behaviours including food stealing, food guarding and aggression both to other dogs and strangers. Further studies are now required to explore the reasons for these associations. 


\section{Acknowledgements}

The authors wish to thank all owners and their dogs for participating in the study, study and to Channel 4 for its help hosting and promoting the survey.

The study was not funded by any research grant. During this study the position of C. W. was funded by the UK Medical Research Council (Population Health Scientist Fellowship grant number G1002402).

Royal Canin financially supports the post of A. J. G. at the University of Liverpool, whilst Dogs Trust financially supports the post of E. B. at the University of Bristol.

A. J. G., E. B. and M. E. proposed the research questions and designed the survey. A. J. G. conducted the statistical analyses and C. W. advised. A. J. G., E. B. and C. W. interpreted the results. A. J. G. produced the first draft of the manuscript, which was subsequently reviewed and edited by the other authors. All authors approved the final manuscript.

\section{References}

1. Buckland EL, Corr SA, Abeyesinghe SM, et al.. (2014) Prioritisation of companion dog welfare issues using expert consensus. Anim Welf 23, 39-46.

2. Vacalopoulos A \& Anderson RK (1993) Canine behaviour problems reported by clients in a study of veterinary hospitals. Appl Anim Behav Sci 37, 84.

3. Wells DL \& Hepper PG (2000) Prevalence of behaviour problems reported by owners of dogs purchased from an animal rescue shelter. Appl Anim Behav Sci 69, 55-65.

4. Lund EM, Armstrong PJ, Kirk CA, et al. (2006) Prevalence and risk factors for obesity in adult dogs from private US veterinary practices. Int J Appl Res Vet Med 4, 177-186.

5. Courcier EA, Thomson RM, Mellor DJ, et al. (2010) An epidemiological study of environmental factors associated with canine obesity. J Small Anim Pract 51, 362-367.

6. Banfield Pet Hospital (2012) State of Pet Health 2012 Report. http://www.stateofpethealth.com/Content/pdf/State_of_Pet_Health_ 2012.pdf
7. German AJ (2006) The growing problem of obesity in dogs and cats. J Nutr 136, 1940S-1946S.

8. German AJ, Holden SL, Wiseman-Orr ML, et al. (2012) Quality of life is reduced in obese dogs but improves after successful weight loss. Vet J 192, 428-434.

9. Kealy RD, Lawler DF, Ballam JM, et al. (2002) Effects of diet restriction on life span and age-related changes in dogs. J Am Vet Med Assoc 220, 1315-1320.

10. German AJ, Blackwell E, Evans M, et al. (2017) Overweight dogs exercise less frequently and for shorter periods: results of a large online survey of $\operatorname{dog}$ owners from the UK. J Nutr Sci 6, doi:10.1017/jns.2017.6.

11. Pickup E, German AJ, Blackwell E, et al. (2017) Variation in activity levels amongst dogs of different breeds: results of a large online survey of $\operatorname{dog}$ owners from the UK. J Nutr Sci 6, doi:10.1017/jns.2017.7.

12. Holm S (1979) A simple sequentially rejective multiple test procedure. Scand J Stat 6, 65-70.

13. Raffan E, Dennis RJ, O’Donovan CJ, et al. (2016) A deletion in the canine POMC gene is associated with weight and appetite in obesity-prone Labrador retriever dogs. Cell Metab 23, 893-900.

14. Hughes SO, Power TG, Orlet Fisher J, et al. (2005) Revisiting a neglected construct: parenting styles in a child-feeding context. Appetite 44, 83-92.

15. Faith MS, Scanlon KS, Birch LL, et al. (2004) Parent-child feeding strategies and their relationships to child eating and weight status. Obes Res 12, 1711-1722.

16. German AJ (2015) Style over substance: what can parenting styles tell us about ownership styles and obesity in companion animals? $\mathrm{Br}$ J Nutr 113, Suppl. 1, S72-S77.

17. Kienzle E, Bergler R \& Mandernach A (1998) Comparison of the feeding behavior of the man-animal relationship in owners of normal and obese dogs. J Nutr 128, 2779S-2782S.

18. Robertson ID (2003) The association of exercise, diet and other factors influence of diet and other factors with owner-perceived obesity in privately owned dogs from metropolitan Perth, Western Australia. Prev Vet Med 58, 75-83.

19. Degeling C \& Rock M (2012) 'It was not just a walking experience': reflections on the role of care in dog-walking. Health Promotion Int 28, 397-406.

20. Eastland-Jones RC, German AJ, Holden SL, et al. (2014) Owner misperception of canine body condition persists despite use of a body condition score chart. J Nutr Sci 3, e45. 